PHYSICAL REVIEW D 94, 044042 (2016)

\title{
From smooth curves to universal metrics
}

\author{
Metin Gürses, ${ }^{1, *}$ Tahsin Çağrı Şişman, ${ }^{2, \dagger}$ and Bayram Tekin ${ }^{3, \sharp}$ \\ ${ }^{1}$ Department of Mathematics, Faculty of Sciences Bilkent University, 06800 Ankara, Turkey \\ ${ }^{2}$ Department of Astronautical Engineering, University of Turkish Aeronautical Association, \\ 06790 Ankara, Turkey \\ ${ }^{3}$ Department of Physics, Middle East Technical University, 06800 Ankara, Turkey
}

(Received 27 June 2016; published 22 August 2016)

\begin{abstract}
A special class of metrics, called universal metrics, solves all gravity theories defined by covariant field equations purely based on the metric tensor. Since we currently lack the knowledge of what the full quantum-corrected field equations of gravity are at a given microscopic length scale, these metrics are particularly important in understanding quantum fields in curved backgrounds in a consistent way. However, finding explicit universal metrics has been a difficult problem as there does not seem to be a procedure for it. In this work, we overcome this difficulty and give a construction of universal metrics of $d$-dimensional spacetime from curves constrained to live in a $(d-1)$-dimensional Minkowski spacetime or a Euclidean space.
\end{abstract}

DOI: 10.1103/PhysRevD.94.044042

\section{INTRODUCTION}

There is a nonignorable problem in high-energy gravity: we do not know the full field equations and the microscopic degrees of freedom responsible for gravity. What we know is that Einstein's theory is an effective one which will be modified with powers of curvature and its derivatives, (most probably) in a diffeomorphism invariant way, as long as the Riemannian spacetime model remains intact as a valid description of gravity. At this stage, there is no compelling reason to suspect that such a description ceases to make sense well below the Planck scale. One might be deterred to say anything about high-energy gravity, in the absence of what the theory is, but the situation is not that bleak as there are certain types of spacetimes that solve any metric-based equations. This approach to high-energy gravity is a remarkable one which started long ago [1,2] not exactly in this language, but developed [3-5] over the years and culminated in a rather nice summary [6], where the notion of universal metrics with further refinements was made, see also the more recent discussion in $[7,8]$. Note that our definition of a universal metric is somewhat different from the one defined in the previous literature: namely, for us, a universal metric is a metric that solves all gravity theories defined by covariant field equations purely based on the metric tensor. (We shall not go into that distinction here and also not distinguish "critical" versus "noncritical" metrics, where the former extremize an action while the latter solve a covariantly conserved field equation not necessarily coming from an action.)

\footnotetext{
*gurses@fen.bilkent.edu.tr tahsin.c.sisman@gmail.com

btekin@metu.edu.tr
}

The interest in the universal metrics is actually two-fold: these are valuable on their own as they are solutions to putative low-energy quantum gravity at any order in the curvature. But, as importantly, when one does quantum field theory at high energies, working about these solutions will provide a better, self-consistent picture as gravity also plays a role. Of course, such metrics are hard to find, as we are not given the equations. Therefore, one will be hardpressed to find, in the literature, examples of these metrics other than the examples given in the papers noted above. More important, perhaps, is the fact that there is really no well-defined procedure for finding these solutions except trial and error: namely, given a rather symmetric metric, one can compute all possible curvature invariants and hope that they vanish or, at best, that they are constants and all conserved second-rank tensors built from the Riemann tensor and its derivatives are proportional to the metric and the Ricci tensor.

In this work, we shall show that there is a proper way to find universal metrics in $d$ dimensions using curves in one less dimension. The generation of this not-so-obvious solution came as a serendipitous surprise in our rather intense excursion to the universal metric territory in the following works: we have shown that the plane wave and spherical wave metrics, built on the anti-de Sitter seeds, solve generic gravity theories [9-11], modulo the assumption that the Lagrangian is solely composed of the curvature, covariant derivatives of the curvature, and the metric tensor in a Lorentz-invariant way (or the field equation is a covariantly conserved two-tensor built from the metric). All of these solutions are in the form of the Kerr-Schild-Kundt metrics,

$$
g_{\mu \nu}=\bar{g}_{\mu \nu}+2 V \lambda_{\mu} \lambda_{\nu}
$$


where the seed $\bar{g}_{\mu \nu}$ metrics are maximally symmetric, whose explicit forms will be dictated by the curves that will generate the solutions. The other ingredients of (1) will be discussed below. We first discuss the curves.

\section{CURVES IN FLAT SPACETIMES}

Let $z^{\mu}(\tau)$ define a smooth curve $C$ in $\mathbb{R}^{d}$, with the metric $\eta_{\mu \nu}$. Here $\tau$ is the parameter of the curve. From an arbitrary point $P\left(x^{\mu}\right)$ not on the curve, there are two null lines intersecting the curve at two points as shown in the Fig. 1. ${ }^{1}$

These intersection points are called the retarded $\left(\tau_{0}\right)$ and advanced $\left(\tau_{1}\right)$ times $[12,13]$. Let $\Omega$ be the distance between the points $P\left(x^{\mu}\right)$ and $Q\left(z^{\mu}\right)$, then since the spacetime is flat, it is simply given as

$$
\Omega^{2}=\eta_{\mu \nu}\left(x^{\mu}-z^{\mu}(\tau)\right)\left(x^{\nu}-z^{\nu}(\tau)\right),
$$

which vanishes for the retarded and advanced times. There is a natural null vector $\partial_{\mu} \tau_{0}$ that one can obtain by differentiating $\Omega\left(\tau_{0}\right)=0$ with respect to $x_{\mu}$ as

$$
\ell_{\mu} \equiv \partial_{\mu} \tau_{0}=\frac{x_{\mu}-z_{\mu}\left(\tau_{0}\right)}{R}
$$

where $R$ is the retarded distance: $R \equiv \dot{z}^{\alpha}\left(\tau_{0}\right)\left(x_{\alpha}-z_{\alpha}\left(\tau_{0}\right)\right)$ with $\dot{z}^{\alpha}\left(\tau_{0}\right) \equiv \partial_{\tau_{0}} z^{\alpha}\left(\tau_{0}\right)$. We have chosen to work with the retarded time $\tau_{0}$, but we could equally have worked with the advanced time $\tau_{1}$ and the ensuing results would not change. Moreover, in what follows, for notational simplicity, we omit the subscript 0 from $\tau_{0}$ and use $\tau$ instead. Taking one more partial derivative of the null vector, one has

$$
\partial_{\nu} \ell_{\mu}=\frac{1}{R}\left(\eta_{\mu \nu}-\dot{z}_{\mu} \ell_{\nu}-\dot{z}_{\nu} \ell_{\mu}-(A-\epsilon) \ell_{\mu} \ell_{\nu}\right),
$$

with $A \equiv \ddot{z}^{\mu}\left(x_{\mu}-z_{\mu}\right)$ and $\epsilon \equiv \dot{z}^{\mu} \dot{z}_{\mu},(\epsilon= \pm 1,0)$, and the argument of $z^{\mu}$ and its derivatives is always the retarded time.

\section{UNIVERSAL METRICS}

The above has been a generic discussion of the curves in flat backgrounds. Now comes the remarkable part of employing these curves to generate solutions of generic gravity theories. Let us assume that the spacetime metric is of the form (1). Then, one can show that the following relations hold for the metrics belonging to the Kerr-SchildKundt class $[9,10]$

$$
\begin{array}{rlrl}
\lambda^{\mu} \lambda_{\mu} & =0, & & \nabla_{\mu} \lambda_{\nu} \equiv \xi_{(\mu} \lambda_{\nu)}, \\
\xi_{\mu} \lambda^{\mu} & =0, & \lambda^{\mu} \partial_{\mu} V=0 .
\end{array}
$$

\footnotetext{
${ }^{1}$ Here, note that we take a generic curve such that it always has at least one intersection with the null cone drawn from an arbitrary point in the Minkowski spacetime.
}

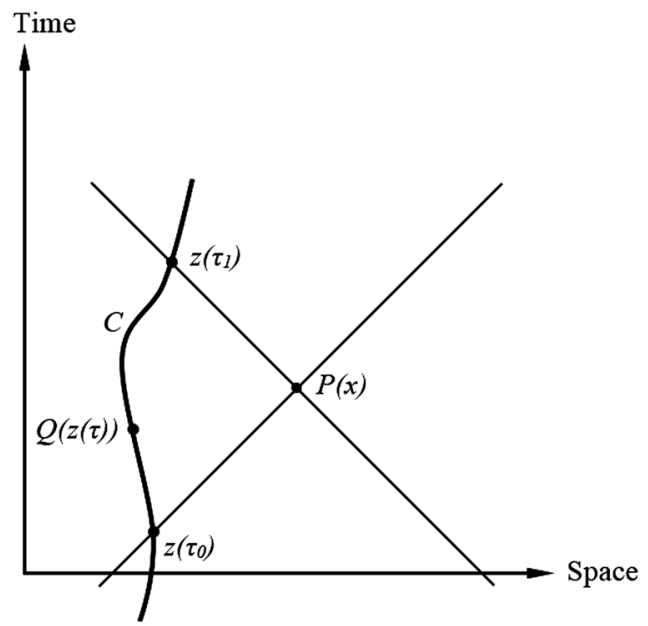

FIG. 1. Two null lines stretching from an arbitrary point $P(x)$ outside the curve meet the curve $C$ at the points corresponding to the retarded and advanced times, that is $\tau_{0}$ and $\tau_{1}$, respectively. $Q(z(\tau))$ represents an arbitrary point on the curve.

It is important to note that a new vector $\xi_{\mu}$ appears, besides the two defining ingredients of the metric, the profile function $V$ and the vector $\lambda_{\mu}$. The first three relations describe Kerr-Schild metrics belonging to the Kundt class and the last relation is an assumption which puts a further restriction on this class of metrics. However, this last relation is crucial in proving the universality of KSK metrics [11]. The covariant derivative of $\lambda_{\mu}$ satisfies $\nabla_{\mu} \lambda_{\nu}=$ $\bar{\nabla}_{\mu} \lambda_{\nu}$ where $\bar{\nabla}_{\mu}$ is the covariant derivative of the seed metric. Then, for the AdS metric in the conformally flat coordinates

$$
d \bar{s}^{2}=\frac{\ell^{2}}{z^{2}}\left(-d t^{2}+\sum_{m=1}^{d-2}\left(d x^{m}\right)^{2}+d z^{2}\right),
$$

$\nabla_{\mu} \lambda_{\nu}$ can be calculated as

$$
\nabla_{\mu} \lambda_{\nu}=\partial_{\mu} \lambda_{\nu}-\frac{1}{z} \eta_{\mu \nu} \lambda_{z}+\frac{1}{z}\left(\lambda_{\mu} \delta_{\nu}^{z}+\lambda_{\nu} \delta_{\mu}^{z}\right)
$$

On the other hand, for the $\mathrm{dS}$ seed metric in the conformally flat coordinates

$$
d \bar{s}^{2}=\frac{\ell^{2}}{t^{2}}\left(-d t^{2}+\sum_{m=1}^{d-1}\left(d x^{m}\right)^{2}\right),
$$

one has

$$
\nabla_{\mu} \lambda_{\nu}=\partial_{\mu} \lambda_{\nu}-\frac{1}{t} \eta_{\mu \nu} \lambda_{t}+\frac{1}{t}\left(\lambda_{\mu} \delta_{\nu}^{t}+\lambda_{\nu} \delta_{\mu}^{t}\right)
$$

By using these results and the defining expression $\nabla_{\mu} \lambda_{\nu}=$ $\xi_{(\mu} \lambda_{\nu)}$ from (5), the partial derivative of $\lambda_{\mu}$ can be written, collectively for the AdS and dS, as 


$$
\partial_{\nu} \lambda_{\mu}=a \eta_{\mu \nu}+\lambda_{\mu}\left(\frac{1}{2} \xi_{\nu}-\zeta_{\nu}\right)+\lambda_{\nu}\left(\frac{1}{2} \xi_{\mu}-\zeta_{\mu}\right)
$$

where $a=\frac{\lambda_{z}}{z}, \zeta_{\nu}=\frac{1}{z} \delta_{\nu}^{z}$ for the AdS seed [9] and $a=-\frac{\lambda_{t}}{t}$, $\zeta_{\nu}=\frac{1}{t} \delta_{\nu}^{t}$ for the dS seed.

The traceless-Ricci tensor, $S_{\mu \nu} \equiv R_{\mu \nu}-\frac{R}{d} g_{\mu \nu}$, and the Weyl tensor, $C_{\mu \alpha \nu \beta}$, can be found, after some tedious computation, as [10]

$$
S_{\mu \nu}=\rho \lambda_{\mu} \lambda_{\nu}, \quad C_{\mu \alpha \nu \beta}=4 \lambda_{[\mu} \Omega_{\alpha][\beta} \lambda_{\nu]},
$$

where the square brackets denote anti-symmetrization with a $1 / 2$ factor and the scalar function $\rho$ is given as

$\rho=-\left(\square+2 \xi^{\mu} \partial_{\mu}+\frac{1}{2} \xi^{\mu} \xi_{\mu}-\frac{2(d-2)}{\ell^{2}}\right) V \equiv-\mathcal{Q} V$.

The second equality defines the operator $\mathcal{Q}$ which will play a role in the field equations of the generic theory below. The symmetric tensor $\Omega_{\alpha \beta}$, that appears in the Weyl tensor, can be compactly written as

$$
\begin{aligned}
\Omega_{\alpha \beta} \equiv & -\left[\nabla_{\alpha} \partial_{\beta}+\xi_{(\alpha} \partial_{\beta)}+\frac{1}{2} \xi_{\alpha} \xi_{\beta}\right. \\
& \left.-\frac{1}{d-2} g_{\alpha \beta}\left(\mathcal{Q}+\frac{2(d-2)}{\ell^{2}}\right)\right] V .
\end{aligned}
$$

For the seed metric, there are three possible choices whose explicit forms are

$$
\begin{aligned}
& d \bar{s}^{2}=\frac{\ell^{2}}{\cos ^{2} \theta}\left(\frac{-d u^{2}+2 d u d r}{r^{2}}+d \theta^{2}+\sin ^{2} \theta d \omega^{2}\right), \\
& d \bar{s}^{2}=\frac{\ell^{2}}{z^{2}}\left(d u^{2}+2 d u d r+d x^{2}+\cdots+d z^{2}\right), \\
& d \bar{s}^{2}=\frac{\ell^{2}}{\cosh ^{2} \theta}\left(\frac{d u^{2}+2 d u d r}{r^{2}}+d \theta^{2}+\sinh ^{2} \theta d \omega^{2}\right),
\end{aligned}
$$

where $\ell$ is related to the cosmological constant and $d \omega^{2}$ is the metric of the $(d-3)$ unit-sphere. The first and the second metrics are AdS metrics, while the third one is the dS metric.

Recently [10], we have shown that the AdS-plane wave and the $p p$-wave metrics in the Kerr-Schild form, and more generally all Kerr-Schild-Kundt metrics are universal. The seed is the flat Minkowski metric for the $p p$-waves, it is the AdS metric for the AdS-plane and AdS-spherical waves, and it is the dS metric for the dS-hyperbolic wave. Referring to $[10,11]$ for the full proof, let us briefly recapitulate how this works.

Let the most general gravity theory be a $(2 N+2)$ derivative theory. As examples, for Einstein's gravity (and Einstein-Gauss-Bonnet gravity) $N=0$, for quadratic and $f$ (Riemann) theories $N=1$, and for higher order theories $N \geq 2$. We have shown that the equations of the most general $(2 N+2)$-derivative gravity theory reduce, when evaluated for these metrics, to a rather compact form

$$
e g_{\mu \nu}+\sum_{n=0}^{N} a_{n} \square^{n} S_{\mu \nu}=0,
$$

where $e$ and $a_{n}$ s are constants which are functions of the parameters of the theory. Here, the constant $e$ determines the possible effective cosmological constants in terms of the parameters of the theory. After some algebraic manipulations, the traceless part of (17) reduces to a scalar equation of the metric function $V$ :

$$
\prod_{n=1}^{N}\left(\mathcal{Q}-m_{n}^{2}\right) \mathcal{Q} V=0 .
$$

The generic solution is $V=V_{E}+\sum_{n=1}^{N} V_{n}$ where the Einsteinian part $\left(V_{E}\right)$ and the other (massive) parts satisfy the following equations, respectively,

$$
\mathcal{Q} V_{E}=0, \quad\left(\mathcal{Q}-m_{n}^{2}\right) V_{n}=0,
$$

provided that all $m_{n}$ 's are different and none is zero. If any two or more $m_{n}$ 's coincide and or equal to zero, then the second equation in (19) changes in the following way: let $r$ be the number (multiplicity) of $m_{n}$ 's that are equal to $m_{r}$, then the corresponding $V_{r}$ satisfies an irreducibly higher derivative equation,

$$
\left(\mathcal{Q}-m_{r}^{2}\right)^{r} V_{r}=0,
$$

with new branches, so-called log solutions, appearing. In that case, the general solution becomes $V=V_{E}+V_{r}+$ $\sum_{n=0}^{N-r} V_{n}$ and $V_{r}$ contains $\log ^{r-1}$ terms.

Let us now get back to the issue of constructing these solutions from the curves in flat space discussed in the previous section. The structural similarity of the partial derivative of $\ell_{\mu}$ in (4) and the partial derivative of $\lambda_{\mu}$ in (10) suggests the following procedure of generating KerrSchild-Kundt class metrics: First, one takes the vectors $\ell_{\mu}$ and $\lambda_{\mu}$ in (4) and (10) to be equal and derives the corresponding vector $\xi_{\mu}$; and secondly, sets $\lambda^{\mu} \xi_{\mu}=0$ to satisfy the third condition in (5) and to obtain the constraint on $z^{\mu}(\tau)$. The second step constrains $z^{\mu}(\tau)$ curves to live in one less dimension.

Let us execute this procedure: when the seed metric is AdS as given in (6), equating (4) and (10), one finds

$$
\xi_{\mu}=-\frac{2}{R}\left(\dot{z}_{\mu}+\frac{1}{2}(A-\epsilon) \lambda_{\mu}\right)+\frac{2}{z} \delta_{\mu}^{z}
$$

To satisfy $\lambda^{\mu} \xi_{\mu}=0$, we must have $\lambda_{z}=\frac{z}{R}$ and $z_{z}=0$. Hence, all these curves live in a $(d-1)$-dimensional Minkowski spacetime. In this case, we have only timelike 
and null curves. We can have spacelike curves, but the metrics generated by these curves are equivalent to the metrics generated from timelike curves via diffeomorphisms and possibly via complex transformations. On the other hand, when the seed metric is the dS metric as given in (8), we find

$$
\xi_{\mu}=-\frac{2}{R}\left(\dot{z}_{\mu}+\frac{1}{2}(A-\epsilon) \lambda_{\mu}\right)+\frac{2}{t} \delta_{\mu}^{t} .
$$

To satisfy $\lambda^{\mu} \xi_{\mu}=0$, we must have $\lambda_{t}=-\frac{t}{R}$ and $z_{t}=0$. Hence, the curve $C$ in this case lives in a $(d-1)$ dimensional Euclidean space where we can have only spacelike curves. Let us turn to some explicit examples.

\section{EXPLICIT EXAMPLES}

We have infinitely many metrics characterized by the curves either in the $(d-1)$-dimensional Minkowski space or the $(d-1)$-dimensional Euclidean space. In the examples below, for the sake of simplicity, we set $d=4$.

Example 1: (Timelike case) Let $z_{\mu}=\tau \delta_{\mu}^{0}$, then $\tau=t \pm r$. Choose $\tau=t-r$, then $R=-r$ and so one finds

$$
\lambda_{\mu}=\left(1, \frac{\vec{x}}{r}\right), \quad \xi_{\mu}=\frac{2}{r}\left(\delta_{\mu}^{0}-\frac{1}{2} \lambda_{\mu}\right)+\frac{2}{z} \delta_{\mu}^{z} .
$$

This gives the AdS-spherical wave solution together with the profile function $V$ solving the corresponding equations. For the explicit form of $V$, see [9].

Example 2: (Null case) If the curve is null, then $\epsilon=0$. Let $z^{\mu}=\tau n^{\mu}$ where $\eta_{\mu \nu} n^{\mu} n^{\nu}=0$, then we arrive at

$$
\begin{aligned}
\tau & =\frac{x^{2}}{2 n_{\mu} x^{\mu}}, \quad x^{2}=\eta_{\mu \nu} x^{\mu} x^{\nu}, \\
\lambda^{\mu} & =\frac{x^{\mu}-\tau n^{\mu}}{R}, \quad R=n_{\mu} x^{\mu}, \\
A & =0, \quad \lambda^{\mu} n_{\mu}=1 .
\end{aligned}
$$

Furthermore, one finds $\xi^{\mu}=-\frac{2}{R} n^{\mu}+\frac{2}{z} \delta_{z}^{\mu}$. Choosing $n^{\mu}=$ $(1,1,0,0)$ and performing a couple of coordinate transformations, one obtains the AdS-plane wave metric

$d s^{2}=\frac{\ell^{2}}{\rho^{2}}\left(2 d \tau d v+d \sigma^{2}+d \rho^{2}\right)+2 V(\tau, \sigma, \rho) d \tau^{2}$.

Example 3: (Spacelike case) When the curve is spacelike, the AdS seed is not allowed. However, the de Sitter seed is possible, i.e., $\bar{g}_{\mu \nu}=\left(\ell^{2} / t^{2}\right) \eta_{\mu \nu}$. Then, for this case, the vector $\xi_{\mu}$ takes the form (22). Let $z^{\mu}=\tau \delta_{x}^{\mu}$, then we find $\tau=x \pm \sqrt{t^{2}-y^{2}-z^{2}}$ and $R=x-\tau=\mp \sqrt{t^{2}-y^{2}-z^{2}}$. Letting $r=\sqrt{t^{2}-y^{2}-z^{2}}$ and choosing the + sign, we get

$$
\lambda_{\mu}=\left(\frac{t}{r}, 1,-\frac{y}{r},-\frac{z}{r}\right) .
$$

Letting $t=r \cosh (\theta), y=r \sinh \theta \cos \phi, z=r \sinh \theta \sin \phi$, the metric takes the form

$$
\begin{aligned}
d s^{2}= & \frac{\ell^{2}}{r^{2} \cosh ^{2} \theta}\left(d u^{2}+2 d u d r+r^{2}\left(d \theta^{2}+\sinh ^{2} \theta d \phi^{2}\right)\right) \\
& +2 V(u, \theta, \phi) d u^{2}
\end{aligned}
$$

for $\lambda_{\mu}=\delta_{\mu}^{0}$ and $\xi_{\mu}=\frac{1}{r} \delta_{\mu}^{0}+2 \tanh (\theta) \delta_{\mu}^{2}$. The above metric is the dS-hyperbolic wave metric given in (16) which was noticed very recently [14].

\section{KSK METRICS IN ROBINSON-TRAUTMAN COORDINATES IN FOUR DIMENSIONS}

The metric form (1) in the coordinates $(t, x, y, z)$ gives a very complicated expression for the operator $\mathcal{Q}$. With this form, it is highly difficult to solve the equations in (19) for the metric function $V$. In addition, we must also satisfy $\lambda^{\mu} \partial_{\mu} V=0$. For this purpose, one should search for new coordinates where both the metric and the operator $\mathcal{Q}$ take simpler forms. Two of the new coordinates are (the natural coordinates) $\tau$ and $R$. They are defined through $\Omega(\tau)=0$ and $R=\dot{z}^{\alpha}(\tau)\left(x_{\alpha}-z_{\alpha}(\tau)\right)$. The coordinate transformation can be given as [15]

$$
x^{\mu}=R \lambda^{\mu}(\tau, \theta, \phi)+z^{\mu}(\tau), \quad \mu=0,1,2,3 .
$$

Here, the null vector $\lambda^{\mu}$ does not depend on the new coordinate $R$ [15]. In these new coordinates, we have

$$
\partial_{R} V=\frac{\partial x^{\mu}}{\partial R} \partial_{\mu} V=\lambda^{\mu} \partial_{\mu} V=0 .
$$

Hence, the metric function is independent of the new coordinate $R$. Furthermore, in the new coordinates, $\lambda_{\mu} d x^{\mu}=d \tau$. Hence, we have

$$
d s^{2}=d \bar{s}^{2}+2 V(\tau, \theta, \phi) d \tau^{2},
$$

where $d \bar{s}^{2}$ is the background line element. The new form of the metric in the new coordinates is called the RobinsonTrautman (RT) metrics.

In four dimensions, to introduce the KSK metrics in the coordinates of RT metrics, we first need the parametrizations of the two-dimensional unit sphere and the twodimensional unit hyperboloid. A parametrization of the two-dimensional unit sphere, $\left(X^{1}\right)^{2}+\left(X^{2}\right)^{2}+\left(X^{3}\right)^{2}=1$, is given by the spherical coordinates:

$$
\begin{aligned}
& X^{1}=\sin \theta \sin \phi, \\
& X^{2}=\sin \theta \cos \phi, \\
& X^{3}=\cos \theta .
\end{aligned}
$$

Similarly, the parametrization of a two-dimensional hyperboloid, $-\left(Y^{0}\right)^{2}+\left(Y^{1}\right)^{2}+\left(Y^{2}\right)^{2}=-1$, is given by 


$$
\begin{aligned}
& Y^{1}=\sinh \theta \sin \phi, \\
& Y^{2}=\sinh \theta \cos \phi, \\
& Y^{0}=\cosh \theta .
\end{aligned}
$$

\section{A. The AdS background}

Following [15], one can write the KSK metrics (1) for the AdS seed in the following form:

$$
\begin{aligned}
d s^{2}= & \frac{1}{f^{2}}\left(H d \tau^{2}+2 d \tau d r+\frac{r^{2}}{P^{2}}\left(d \theta^{2}+\sin ^{2} \theta d \phi^{2}\right)\right) \\
& +2 V(\tau, \theta, \phi) d \tau^{2},
\end{aligned}
$$

where the metric functions are

$$
\begin{gathered}
H=\epsilon-2 r \partial_{\tau} \log P, \quad f=\frac{r}{\ell P} \cos \theta, \\
P=-\dot{z}^{0}(\tau)+\dot{z}^{1}(\tau) X^{1}+\dot{z}^{2}(\tau) X^{2}, \\
\epsilon=-\left(\dot{z}^{0}(\tau)\right)^{2}+\left(\dot{z}^{1}(\tau)\right)^{2}+\left(\dot{z}^{2}(\tau)\right)^{2}+\left(\dot{z}^{3}(\tau)\right)^{2} .
\end{gathered}
$$

Here, $z^{\mu}=\left(z^{0}(\tau), z^{1}(\tau), z^{2}(\tau), z^{3}(\tau)\right)$ is the parametrization of an arbitrary curve $C$ satisfying (36) with $\epsilon=-1,0$, 1 , and $X^{i}$ 's $(i=1,2,3)$ are defined in (31). As a result of the discussion above, the curve $z(\tau)$ lives in one less dimension since $z^{3}(\tau)=0$. The Ricci tensor takes the form

$$
R_{\mu \nu}=-\frac{3}{\ell^{2}} g_{\mu \nu}+\rho \lambda_{\mu} \lambda_{\nu},
$$

where $\lambda_{\mu}=\delta_{\mu}^{0}$ and the function $\rho$ has the form given in (12). To calculate $\rho$ explicitly, one needs to find $\xi_{\mu}$ from its defining relation:

$$
\nabla_{\mu} \lambda_{\nu}=\bar{\nabla}_{\mu} \lambda_{\nu}=\xi_{(\mu} \lambda_{\nu)}
$$

Here, $\bar{\nabla}$ is the covariant derivative of the AdS seed which can be put in the form

$$
d \bar{s}^{2}=\frac{1}{f^{2}}\left(H d \tau^{2}+2 d \tau d r\right)+\frac{\ell^{2}}{\cos ^{2} \theta} g_{m n} d y^{m} d y^{n},
$$

with the metric of the two-dimensional unit sphere $g_{m n}$. Since $\lambda_{\mu}=\delta_{\mu}^{0}$, one has

$$
\bar{\nabla}_{\mu} \lambda_{\nu}=-\bar{\Gamma}_{\mu \nu}^{\alpha} \lambda_{\alpha}=-\bar{\Gamma}_{\mu \nu}^{0},
$$

and from this relation, $\xi_{\mu}$ can be calculated as

$$
\xi_{\mu}=2 \partial_{\mu} \log f-2 \delta_{\mu}^{r} \partial_{r} \log f+\frac{1}{2} \lambda_{\mu} f^{2} \partial_{r}\left(\frac{H}{f^{2}}\right) .
$$

Using this result in (12), after a long calculation, the function $\rho$ is found to be

$$
\begin{aligned}
\rho= & -\mathcal{Q} V=-\left(\bar{g}^{m n} \bar{\nabla}_{m} \partial_{n}+2 \bar{g}^{m n} \partial_{m} \log f \partial_{n}\right. \\
& \left.+2 \bar{g}^{m n} \partial_{m} \log f \partial_{n} \log f-\frac{4}{\ell^{2}}\right) V,
\end{aligned}
$$

where $\bar{g}_{m n} \equiv \frac{\ell^{2}}{\cos ^{2} \theta} g_{m n}$. Clearly, the operator $\mathcal{Q}$ contains derivatives only with respect to the angular coordinates and can be found once one has the explicit form of the curve given. Then one can solve the massless and massive wave equations given in (19).

\section{B. The dS background}

Since the dS case is similar to the AdS case (albeit with subtle differences) above, without much ado let us give the metric and the relevant results. First, the KSK metrics (1) for the $\mathrm{dS}$ seed become

$$
\begin{aligned}
d s^{2}= & \frac{1}{f^{2}}\left(H d \tau^{2}+2 d \tau d r+\frac{r^{2}}{P^{2}}\left(d \theta^{2}+\sinh ^{2} \theta d \phi^{2}\right)\right) \\
& +2 V(\tau, \theta, \phi) d \tau^{2}
\end{aligned}
$$

where the metric functions are

$$
\begin{gathered}
H=1-2 r \partial_{\tau} \log P, \quad f=\frac{r}{\ell P} \cosh \theta, \\
P=\dot{z}^{1}(\tau) Y^{1}+\dot{z}^{2}(\tau) Y^{2}+\dot{z}^{3}(\tau), \\
1=\left(\dot{z}^{1}(\tau)\right)^{2}+\left(\dot{z}^{2}(\tau)\right)^{2}+\left(\dot{z}^{3}(\tau)\right)^{2} .
\end{gathered}
$$

Here, $Y^{i}$ s $(i=1,2)$ are defined in (32) and $z^{0}(\tau)=0$. The Ricci tensor takes the form

$$
R_{\mu \nu}=\frac{3}{\ell^{2}} g_{\mu \nu}+\rho \lambda_{\mu} \lambda_{\nu}
$$

where $\lambda_{\mu}=\delta_{\mu}^{0}$. The dS seed can be put in the form

$$
d \bar{s}^{2}=\frac{1}{f^{2}}\left(H d \tau^{2}+2 d \tau d r\right)+\frac{\ell^{2}}{\cosh ^{2} \theta} g_{m n} d y^{m} d y^{n},
$$

with the metric of the two-dimensional unit hyperboloid $g_{m n}$. Again, to find the function $\rho$, one needs to find $\xi_{\mu}$ which takes the same form (41). Then the function $\rho$ in (12) becomes

$$
\begin{aligned}
\rho= & -\left(\bar{g}^{m n} \bar{\nabla}_{m} \partial_{n}+2 \bar{g}^{m n} \partial_{m} \log f \partial_{n}\right. \\
& \left.+2 \bar{g}^{m n} \partial_{m} \log f \partial_{n} \log f+\frac{4}{\ell^{2}}\right) V,
\end{aligned}
$$

where $\bar{g}_{m n} \equiv \frac{\ell^{2}}{\cosh ^{2} \theta} g_{m n}$. 


\section{CONCLUSION}

We have given a way of constructing the Kerr-SchildKundt type of metrics which we have shown previously to be universal metrics of generic purely metric-based theories of gravity. It is highly interesting that we have three families of curves generating all these universal metrics. When the seed metric is the AdS spacetime, we have two families corresponding to timelike and null curves. They generate the AdS-plane wave and AdS-spherical wave families. For the dS seed metric, only the spacelike curves generate the dS-hyperbolic wave family. Hence, we obtain, in principle, an infinite number of the Kerr-Schild-Kundt type of metrics where the AdS-plane wave, AdS-spherical wave [9], and dS-hyperbolic wave metrics [14] correspond to the straight lines and the rest of the family offers an exciting new territory of investigation. Using the RobinsonTrautman coordinates, we recast the KSK metrics in a convenient form which is suitable for studying explicit solutions.

\section{ACKNOWLEDGMENTS}

This work is partially supported by TUBITAK. M. G. and B.T. are supported by the TUBITAK Grant No. 113F155. T.C.S. is supported by the Science Academy's Young Scientist Program (BAGEP 2015).
[1] G. W. Gibbons, Commun. Math. Phys. 45, 191 (1975).

[2] S. Deser, J. Phys. A 8, 1972 (1975).

[3] R. Guven, Phys. Lett. B 191, 275 (1987).

[4] G. T. Horowitz and A. R. Steif, Phys. Rev. Lett. 64, 260 (1990).

[5] A. A. Coley, Phys. Rev. Lett. 89, 281601 (2002).

[6] A. A. Coley, G. W. Gibbons, S. Hervik, and C. N. Pope, Classical Quantum Gravity 25, 145017 (2008).

[7] S. Hervik, V. Pravda, and A. Pravdova, Classical Quantum Gravity 31, 215005 (2014).

[8] S. Hervik, T. Malek, V. Pravda, and A. Pravdova, Classical Quantum Gravity 32, 245012 (2015).

[9] I. Güllü, M. Gürses, T. C. Şişman, and B. Tekin, Phys. Rev. D 83, 084015 (2011); M. Gürses, T. C. Şişman, and B. Tekin, Phys. Rev. D 86, 024001 (2012); 86, 024009 (2012).
[10] M. Gürses, S. Hervik, T. C. Şişman, and B. Tekin, Phys. Rev. Lett. 111, 101101 (2013).M. Gürses, T. C. Şişman, and B. Tekin, Phys. Rev. D 90, 124005 (2014); 92, 084016 (2015).

[11] M. Gurses, T. C. Sisman, and B. Tekin, arXiv:1603.06524.

[12] W. B. Bonnor and P.C. Vaidya, in General Relativity, papers in honor of J. L. Synge, edited by L. O. Raifeartaigh (Dublin Institute for Advanced Studies, Dublin, 1972), p. 119.

[13] M. Gürses and O. Sarioglu, Classical Quantum Gravity 19, 4249 (2002); 20, 351 (2003); Gen. Relativ. Gravit. 36, 403 (2004).

[14] M. Gürses, C. Senturk, T. C. Şişman, and B. Tekin, Hyperbolic-dS Plane Waves of Generic Gravity Theories (to be published).

[15] E. T. Newman and T. W. J. Unti, J. Math. Phys. (N.Y.) 4, 1467 (1963). 